\title{
Maternal Serum Angiogenic Factor sFlt-1 to PIGF Ratio in Preeclampsia: A Useful Marker for Differential Diagnosis and Prognosis Evaluation in Chinese Women
}

\author{
Wei-zhen Lou $\mathbb{D}$, Fang Jiang, Jing Hu, Xiao-xu Chen, Ying-na Song, Xi-ya Zhou, Jun-tao Liu, \\ Xu-ming Bian, and Jin-song Gao $\mathbb{C}$ \\ Department of Obstetrics \& Gynecology, Peking Union Medical College Hospital, Peking Union Medical College, Chinese Academy of \\ Medical Sciences, Beijing 100730, China \\ Correspondence should be addressed to Jin-song Gao; gaojingsong@pumch.cn
}

Received 4 February 2019; Revised 30 May 2019; Accepted 19 June 2019; Published 16 July 2019

Academic Editor: Małgorzata Knaś

Copyright (C) 2019 Wei-zhen Lou et al. This is an open access article distributed under the Creative Commons Attribution License, which permits unrestricted use, distribution, and reproduction in any medium, provided the original work is properly cited.

\begin{abstract}
The ratio of soluble fms-like tyrosine kinase-1 to placental growth factor (sFlt-1/PlGF) is elevated and proved to be useful in preeclampsia (PE) diagnosis. Its value in differential diagnosis with other pregnancy complications and prediction of pregnancy duration has yet to be clarified in Chinese population. We retrospectively analyzed 118 singleton pregnancies with suspected or diagnosed PE at the Peking Union Medical College Hospital (PUMCH) in China. Among these, 62 pregnancies were diagnosed as PE (48 early onsets and 14 late onsets, with 39 and 5 severe PE, respectively), 12 gestational hypertension (GH), 15 chronic hypertension (chrHTN), 16 autoimmune diseases, and 13 pregnancies with uncomplicated proteinuria. And 76 normal pregnancies were included as control. The results showed (1) the sFlt-1/PlGF ratio in early onset PE subgroup was significantly higher than that in GH, chrHTN, and control groups; the sFlt-1/PlGF ratio in late onset PE subgroup was significantly higher than that in chrHTN and control groups, but similar as GH group; the sFlt-1/PlGF ratio was similar among GH, chrHTN, and control groups. (2) The sFlt-1/PlGF ratio was significantly increased in the PE group compared with autoimmune disease and uncomplicated proteinuria pregnancies. (3) By ROC curve analysis, the cutoff value of the sFlt-1/PlGF ratio was less than 21.5 to rule out PE and higher than 97.2 to confirm the diagnosis of PE. (4) The sFlt-1/PlGF ratio was higher in PE pregnancies delivering within 7 days than those more than 7 days, either in early onset PE or severe PE. In conclusion, we show that maternal sFlt-1/PlGF ratio is an efficient biomarker in the diagnosis and differential diagnosis of PE. This ratio can be used to predict the timing of delivery for PE pregnancies.
\end{abstract}

\section{Introduction}

Preeclampsia (PE) affects 5 8\% of pregnancies worldwide and remains as a major cause of morbidity and mortality in both the mother and the fetus worldwide [1-4]. PE is of special relevance in the developing countries, where the maternal mortality is $\sim 15 \%$ compared with $0 \sim 1.8 \%$ in the developed countries [5]. In China, diagnosis of PE is mostly based on limited clinical signs and unspecific laboratory findings, which are compromised to make early diagnosis and proper management. Sometimes, it is difficult to differentiate PE from predisposing conditions, such as chronic hypertension, CKD (chronic kidney disease), and autoimmune dis- eases like systemic lupus erythematosus which are also the leading risk factors for PE. Meanwhile, the outcome of PE is hard to predict due to diverse disease progression in individuals [6]. It is valuable to find out a specific marker that can improve diagnostic accuracy, help determine the timing of delivery, and thus improve the outcome of PE.

In the last decade, our understanding of the pathogenesis underlying PE has progressively advanced and it is generally appreciated that early and late onset PE have different pathophysiologies [7-11]. Early onset PE, also referred to as placental PE, arises from a placenta that is under hypoxic conditions with oxidative stress [12], while late onset PE, also called maternal $\mathrm{PE}$, arises from the interaction between a 
normal placenta and a maternal constitution that is susceptible to, or suffers from, metabolic and vascular diseases, as with long-term hypertension or diabetes $[13,14]$. Mixed presentations, combining maternal and placental contributions, are common [15]. Placental PE is characterized by defective deep trophoblastic invasion and impaired maternal spiral artery remodeling in the first half of pregnancy, leading to inadequate placental perfusion in the second half. The state of placental insufficiency triggers an imbalance in the placental release of angiogenesis regulatory factors to the maternal circulation, characterized by decreased concentrations of proangiogenic factors such as placental growth factor (PlGF) and elevated concentrations of prohypertensive and antiangiogenic factors such as soluble fms-like tyrosine kinase-1 (sFlt-1) [16, 17]. PlGF, a member of the VEGF family, can promote placenta angiogenesis, increase vascular permeability, and enhance trophoblast cell activity. Soluble fms-like tyrosine kinase-1 (sFlt-1) can decrease the serum concentration of PlGF, inhibit the biological function of PlGF, and impair the permeability and integrity of vascular wall, leading to angiogenesis disorders, edema, urine protein, and hemoconcentration [18, 19]. Increasing evidences showed that there were elevated serum concentration of sFlt-1 and declined serum concentration of PlGF in PE patients and that the degree of elevated or declined level was correlated with the severity of PE [20]. Because of this, sFlt-1 and PlGF were considered as the most promising serological indicators for PE diagnosis. Particularly, the sFlt-1/PlGF ratio could better reflect the antiangiogenic activity and could be used to predict the occurrence and prognosis of PE [20-22]. However, cutoff values and predictive efficacy varied largely across study countries, suggesting it may not necessarily be similar in different ethnic and geographical populations [23-27]. There were few such studies in Chinese population, and its value in differential diagnosis with other similar pregnancy complications and prediction of pregnancy duration has yet to be clarified.

The objectives of this study are, firstly, to compare the sFlt-1 to the PlGF ratio among PE, other pregnancy complications, and normal controls; secondly, to determine a possible cutoff value aiding to make PE diagnosis; and lastly, to clarify the value of this ratio in the prediction of pregnancy duration in Chinese population.

\section{Materials and Methods}

2.1. Study Population. A hundred and eighteen singleton pregnancies with suspected or diagnosed PE after twenty weeks of gestation were enrolled in our study from Feb 2013 to Nov 2016, who underwent regular maternity examinations in our hospital. Maternal blood was collected and serum was frozen and stored at $-80^{\circ} \mathrm{C}$ for analysis. They were closely monitored and treated according to clinical routines. Final diagnosis was confirmed after birth for each patient. The study was approved by the Ethic Committee of Peking Union Medical College Hospital. Written informed consents were obtained for all the women agreeing to use their banked serum for research purposes.
The diagnosis of gestational hypertensive disorders was defined and classified according to the ACOG definition [28]. PE was defined as hypertension (blood pressure $\geq 140 \mathrm{mmHg}$ systolic and/or $\geq 90 \mathrm{mmHg}$ diastolic) on two separate occasions at least 4 hours apart at or after 20 weeks of gestation accompanied by proteinuria ( 24 hour urine protein $\geq 0.3$ g, spot urine protein/creatinine ratio $(\mathrm{PCr}) \geq 0.3$, or spot urine dipstick $\geq$ " $2+$ ") or evidence of end-organ dysfunction (i.e., thrombocytopenia, severe headache, renal insufficiency, impaired liver function, and heart and lung dysfunction). Chronic hypertension (chrHTN) was defined as hypertension that occurred in $<20$ weeks of gestation and lasted $\geq 12$ weeks after delivery without proteinuria. Gestational hypertension $(\mathrm{GH})$ was defined as hypertension that occurred after 20 weeks of gestation without proteinuria or end-organ dysfunction.

Severe PE refers to PE that reaches any of the following criteria [29-31]: (1) persisting elevated severe hypertension: blood pressure $\geq 160 \mathrm{mmHg}$ systolic and/or $\geq 110 \mathrm{mmHg}$ diastolic; (2) neurological complications (examples include but not restricted to persistent severe headaches and visual disturbances); (3) persistent right upper quadrant or epigastric abdominal pain; (4) elevated transaminases (e.g., ALT or AST); (5) renal function insufficiency: proteinuria $>2.0 \mathrm{~g} / \mathrm{L}$, oliguria (24-hour urine less than $400 \mathrm{~mL}$ or urine per hour less than $17 \mathrm{~mL}$ ), or serum creatinine $>106 \mu \mathrm{mol} / \mathrm{L}$; (6) hypoproteinemia accompanied by ascites, hydrothorax, or hydropericardium; (7) hematological complications (thrombocytopenia-platelet count below $100,000 / \mu \mathrm{L}$, hemolysis manifested by anemia, and elevated lactate dehydrogenase level and jaundice); (8) heart failure; (9) pulmonary edema; and (10) uteroplacental dysfunction (such as fetal growth restriction, oligohydramnios, placenta abruption, or stillbirth).

Delivery plan was based upon maternal and fetalplacental conditions [32]: (1) GH and PE that do not deteriorate to severe $\mathrm{PE}$ were managed conservatively until 37 gestational weeks; (2) offer birth to women with severe PE after 34 weeks when their blood pressure has been controlled and a course of corticosteroids has been completed; (3) offer birth to women with severe PE before 34 weeks (with a course of corticosteroids if possible) if severe hypertension develops refractory to treatment or maternal or fetal's adverse complications develops.

PE patients were classified as early onset ( $<34$ weeks of gestation) or late onset ( $\geq 34$ weeks of gestation) at the time when the diagnosis of $\mathrm{PE}$ was made. Remaining duration of pregnancy refers to the period from enrollment to delivery.

2.2. sFlt-1 and PlGF Assays. Automated assays for sFlt-1 and PlGF (with both levels measured in picograms per milliliter) were performed using the fully automated Elecsys assays on an electrochemiluminescence immunoassay platform (cobas E 601 immunoassay analyzers, Roche Diagnostics) [33]. The interassay coefficient of variance for sFlt- 1 and PlGF immunoassays ranged from 2.6 to $3.0 \%$ and 2.0 to $2.4 \%$, respectively. Test results were blinded to both patients and their physicians.

2.3. Statistical Analysis. The sFlt-1/PlGF ratio (value of sFlt-1 measured in $\mathrm{pg} / \mathrm{mL}$, divided by the value of PlGF measured 
TABLE 1: Baseline characteristics of study population.

\begin{tabular}{|c|c|c|c|c|c|c|}
\hline & Controls & $\mathrm{PE}$ & $\mathrm{GH}$ & chrHTN & CTD & Proteinuria \\
\hline Total $(n)$ & 76 & 62 & 12 & 15 & 16 & 13 \\
\hline Age (y) & $29 \pm 3$ & $31 \pm 5$ & $33 \pm 5$ & $31 \pm 5$ & $31 \pm 5$ & $33 \pm 4$ \\
\hline Multipara (\%) & 18.4 & 28.6 & 16.6 & 13.3 & 0 & 16.7 \\
\hline Gestational week of test & $30 \pm 5$ & $31 \pm 4$ & $33 \pm 3$ & $32 \pm 4$ & $32 \pm 4$ & $30 \pm 4$ \\
\hline Gestational week of delivery & $39 \pm 2$ & $32 \pm 4^{\mathrm{a}}$ & $38 \pm 2$ & $39 \pm 1$ & $37 \pm 3$ & $39 \pm 1$ \\
\hline Birthweight (g) & $3198 \pm 388$ & $1624 \pm 703^{\mathrm{a}}$ & $3130 \pm 584$ & $3167 \pm 399$ & $2639 \pm 616$ & $3080 \pm 325$ \\
\hline sFlt-1/PlGF ratio & $4.1 \pm 0.5$ & $257.7 \pm 44.7^{\mathrm{a}}$ & $38.6 \pm 17.4$ & $8.9 \pm 2.4$ & $19.6 \pm 6.9$ & $10.5 \pm 3.5$ \\
\hline
\end{tabular}

${ }^{a}$ Women with PE had a lower mean birthweight of the neonate, a shorter mean gestational week of delivery, and a higher sFlt-1/PlGF ratio than healthy controls or women with GH, chrHTN, CTD, or proteinuria. The difference was significant, $P<0.001$.

Table 2: sFlt-1/PlGF ratio in PE, GH, chrHTN, and healthy controls.

\begin{tabular}{|c|c|c|c|c|c|c|c|c|}
\hline \multirow{2}{*}{ Group } & \multicolumn{2}{|c|}{$\mathrm{PE}(n=62)$} & \multicolumn{2}{|c|}{ Control $(n=76)$} & \multicolumn{2}{|c|}{$\mathrm{GH}(n=12)$} & \multicolumn{2}{|c|}{$\operatorname{chrHTN}(n=15)$} \\
\hline & $<34$ wks & $\geq 34$ wks & $<34$ wks & $\geq 34$ wks & $<34$ wks & $\geq 34$ wks & $<34$ wks & $\geq 34$ wks \\
\hline Total $(n)$ & 48 & 14 & 59 & 17 & 5 & 7 & 10 & 5 \\
\hline sFlt-1/PlGF ratio & $311.9 \pm 55.4$ & $71.7 \pm 11.7$ & $2.9 \pm 0.2$ & $8.3 \pm 1.7$ & $21.5 \pm 18.8$ & $50.8 \pm 26.9$ & $8.1 \pm 3.0$ & $10.4 \pm 4.1$ \\
\hline$P$ value $^{\mathrm{a}}$ & \multicolumn{2}{|c|}{$<0.001^{\mathrm{a}}$} & \multicolumn{2}{|c|}{$0.005^{\mathrm{a}}$} & \multicolumn{2}{|c|}{0.432} & \multicolumn{2}{|c|}{0.665} \\
\hline
\end{tabular}

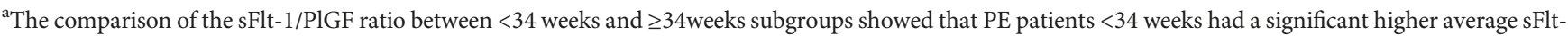
$1 /$ PIGF ratio than patients $\geq 34$ weeks; controls $<34$ weeks significantly decreased than controls $\geq 34$ weeks.

in $\mathrm{pg} / \mathrm{mL}$ ) was used as a measure of circulating angiogenic imbalance. Box-whisker plots were generated to compare the sFlt-1/PlGF ratio between groups. Baseline characteristics and the sFlt-1/PlGF ratio of patient groups were compared using the $t$-test and the chi-squared test which were appropriate. Independent $t$-test was used for normally distributed continuous variables and the chi-squared test $\left(\chi^{2}\right)$ for categorical variables. Variance analysis was applied to examine differences in the main predictors of interests. When the variance was homogenous, the Bonferroni (B) test was performed; otherwise, Tamhane's T2 (M) test was performed. We also used the receiver operating characteristic (ROC) analysis to determine the cutoff value of the sFlt-1/PlGF ratio in the diagnosis of PE. The sensitivity, specificity, and positive and negative predictive values of the cutoff value were calculated.

All $P$ values reported were 2 -tailed. The $P$ values of $<0.05$ were considered statistically significant. All statistical analyses were performed with the use of Statistical Package for Social Sciences (SPSS, version 22.0).

\section{Results}

3.1. Clinical Features of the Study Population. A total of 118 singleton pregnancies included $62 \mathrm{PE}$ (48 early onsets and 14 late onsets, with 39 and 5 severe PE, respectively), 12 GH, 15 chrHTN, 16 CTD (13 systemic lupus erythematosus, one Sjogren's syndrome, one undifferentiated connective tissue disease, and one antiphospholipid syndrome), and 13 uncomplicated proteinuria. For the control group, 76 normal singleton pregnancies were included. There were no significant differences in maternal age, parity, and gestational week of enrollment among groups. Women with PE had a lower neonate birth weight (1624 $\pm 704 \mathrm{~g})$ and an earlier gestational week of delivery $(32 \pm 4 \mathrm{w})$ compared with healthy control $(3198 \pm 388 \mathrm{~g}, 39 \pm 2 \mathrm{w})$ or women with $\mathrm{GH}$, chrHTN, CTD, or uncomplicated proteinuria $(P<0.001)$ (Table 1$)$.

3.2. sFlt-1/PlGF Ratio in Different Groups. The sFlt-1/PlGF ratio was higher in $\mathrm{PE}$ patients $(257.7 \pm 44.7)$ than in normal control $(4.1 \pm 2.5)$ or other groups (GH, chrHTN, CTD, and uncomplicated proteinuria patients, with mean level ranged from 8.9 to $38.6, P<0.001)$. There was no difference between other groups and healthy control (Table 1).

Early onset PE had higher sFlt-1/PlGF ratio compared with late onset $\mathrm{PE}(311.9 \pm 55.4$ vs. $71.7 \pm 11.7, P<0.001)$. In the window of $<34$ weeks of gestation (early onset), the average Flt-1/PlGF ratio was significantly higher in $\mathrm{PE}$ patients than in $\mathrm{GH}$, chrHTN, or healthy control $(P<0.001$ ). In the window of $\geq 34$ weeks of gestation, the average ratio in $\mathrm{PE}$ is also increased $(P=0.001)$ but the difference between $\mathrm{PE}$ and $\mathrm{GH}$ groups did not reach statistical significance $(71.7 \pm 11.7$ vs. $50.8 \pm 26.9, P=0.984)$ (Table 2). Patients with $\mathrm{GH}$ or chrHTN had a slightly higher sFlt-1/PlGF ratio either before or after 34 weeks of gestation compared with the control group, but this difference did not reach statistical significance (Table 2 and Figure 1).

Severe PE patients $(44 / 62,314.2 \pm 60.0)$ had higher sFlt$1 / \mathrm{PlGF}$ ratio than the mild ones $(18 / 62,119.5 \pm 29.2, P=$ $0.005)$. At $<34$ weeks, the sFlt-1/PlGF ratio in severe $\mathrm{PE}$ patients was higher than mild patients, but the difference was not significant $(344.7 \pm 66.1$ vs. $169.9 \pm 53.0, P=0.221)$. After 34 weeks, the average ratios of severe and mild PE were similar $(76.1 \pm 24.2$ vs. $69.2 \pm 13.5, P=0.791)$.

3.3. Cutoff Value of sFlt-1/PlGF Ratio in the Diagnosis of PE. In order to determine the performance of the sFlt-1/PlGF ratio in clinical settings, ROC curves were constructed. The sFlt-1/PlGF ratio had an AUC of 0.98 (95\% CI, 0.969- 


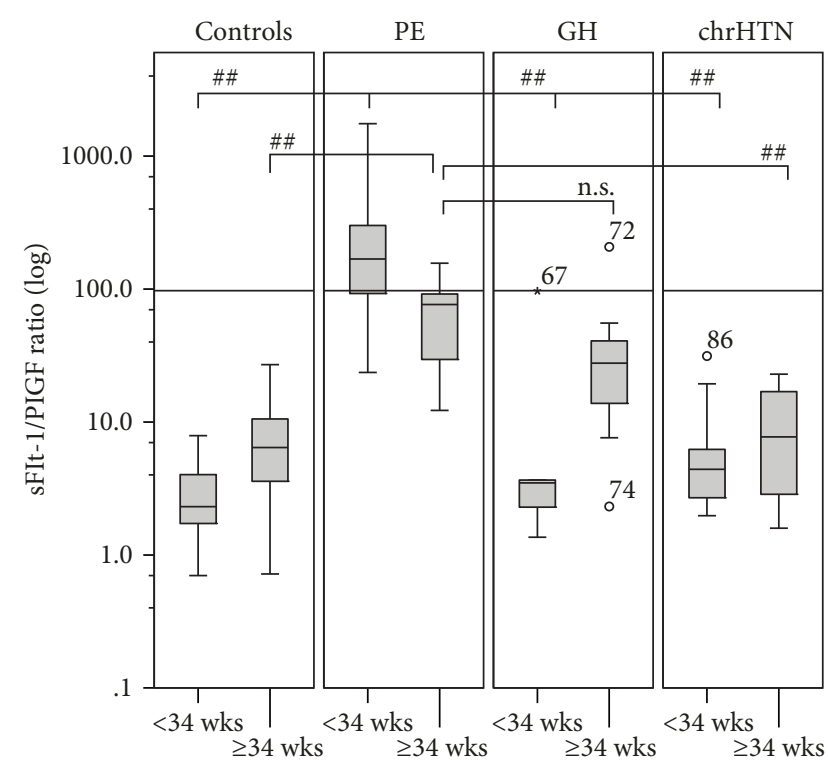

(a)

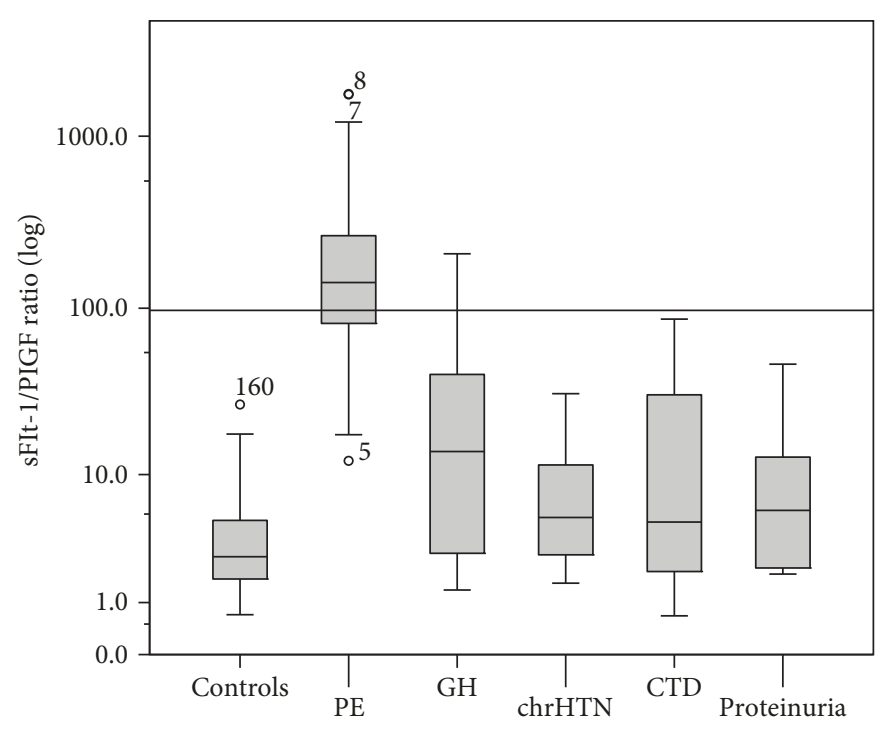

(b)

FIGURE 1: sFlt-1/PlGF ratio in PE, GH, chrHTN, CTD, proteinuria, and controls. Box-whisker plots displayed the sFlt-1/PlGF ratio in patients with $\mathrm{PE}, \mathrm{GH}$, chrHTN, pregnancies with CTD or proteinuria, and controls. Boxes indicate interquartile range; whisker indicates range; error bars indicate median. O means mild outliers. ${ }^{*}$ means extreme outliers. The cutoff of 97.2 was drawn into the graph as a solid line. (a) The logarithmic distribution of the sFlt-1/PlGF ratio in patients with PE/GH/chrHTN/controls. The average Flt-1/PlGF ratio was significantly higher in PE $<34$ weeks $(n=48)$ than in GH $(n=5)$, chrHTN $(n=10)$, or controls $(n=59)<34$ weeks $($ all $P<0.001)$. PE $\geq 34$ weeks $(n=14)$ was compared with chrHTN $(n=5)$ and controls $(n=17) \geq 34$ weeks (all $P=0.001)$, though the difference did not reach statistical significance between $\mathrm{PE}$ and $\mathrm{GH} \geq 34$ weeks $(n=7)(P=0.984)$. (b) The logarithmic distribution of the sFlt-1/PlGF ratio in patients with $\mathrm{PE}, \mathrm{GH}$, chrHTN, pregnancies with CTD or proteinuria, and controls. The sFlt-1/PlGF ratio was higher in PE than in GH, chrHTN, CTD $(n=16)$, proteinuria $(n=13)$, and controls (all $P<0.001)$, while no significant differences between any other two groups.

1.000), 0.99 (95\% CI, 0.989-1.000), and 0.91 (95\% CI, 0.822$1.000)$ for all PE, early onset PE ( $<34$ weeks), and late onset $\mathrm{PE}$ ( $\geq 34$ weeks), respectively (Figure 2 ).

The sFlt-1/PIGF ratio of 21.5 had the best performance to rule out PE before 34 weeks of gestation, with the NPV of $97.9 \%, 100 \%$, and $91.7 \%$ for all, early onset, and late onset PE. Meanwhile, the value of 97.2 had the best performance to confirm the diagnosis of $\mathrm{PE}$ before 34 weeks, with the PPV of $97.5 \%, 100 \%$, and $75.0 \%$ for all, early onset, and late onset PE. This result confirmed the potential clinical usage of the sFlt-1/PIGF ratio for the diagnosis of PE especially before 34 weeks of gestation.

If we utilize the reported cutoff value [6] in our Chinese cohort, using 85 for early onset PE and 111 for late onset PE to confirm diagnosis, the PPV value will be $95.7 \%$, 97.4\%, $85.7 \%$ and $97.4 \%, 100.0 \%, 75.0 \%$ accordingly. These results suggest that the reported value is also feasible in our population.

3.4. sFlt-1/PlGF Ratio and Remaining Duration of Pregnancy. As time to delivery is an essential indicator of disease severity, we divided the $62 \mathrm{PE}$ patients into two subgroups: delivered within 7 days (45/62) or longer than 7 days (17/62) from the time of enrollment. The sFlt-1/PlGF ratio in the subgroup delivered within $7 \mathrm{~d}$ was significantly higher than the subgroup delivered longer than $7 \mathrm{~d}(309.4 \pm 59.6$ vs. $120.9 \pm 18.0, P=0.004)$. We also compared the differences between the two subgroups in early onset (48/62), severe
(44/62), or early onset severe PE patients (39/62). The sFlt-1/PlGF ratios in subgroups delivered within $7 \mathrm{~d}$ were also significantly higher than that in subgroups longer than $7 \mathrm{~d}$ whether it is early onset $\mathrm{PE}(369.3 \pm 71.1$ vs. $139.8 \pm$ 21.8, $P=0.004)$, severe PE $(372.4 \pm 74.7$ vs. $116.4 \pm 25.1$, $P=0.002)$, or early onset severe PE ( $409.8 \pm 82.3$ vs. 128.0 $\pm 24.9, P=0.002)$. There were no significant differences between the two subgroups in late onset $\mathrm{PE}(69.6 \pm 13.9$ vs. $75.4 \pm 23.3, P=0.822)$ or mild PE (114.6 \pm 45.6 vs. $127.2 \pm$ 27.3, $P=0.841$ ) (Figure 3).

The third quartile (Q3) of the sFlt-1/PlGF ratio was 301.0 in early onset PE patients. Of the 48 early onset preeclampsia patients, 12 patients (12/48) had sFlt-1/PlGF ratio greater than Q3, all of them delivered within 7 days. In the 36 patients (36/48) whose Flt-1/PlGF ratio were below Q3, $24(24 / 36,66.7 \%)$ were delivered within 7 days (100\% vs. $66.7 \%, P=0.021$ ). One early onset mild PE patient who had a Flt-1/PlGF ratio greater than Q3 was delivered 4 days after recruitment. None of the late onset patient had a ratio higher than 301.0.

\section{Discussion}

4.1. The sFlt-1/PlGF Ratio Is Useful in the Diagnosis and Differential Diagnosis of PE. The diagnosis of PE is mainly based on clinical manifestations and unspecific biochemical makers. Some patients with certain complications like chrHTN and CTD are doomed to progress into PE while 


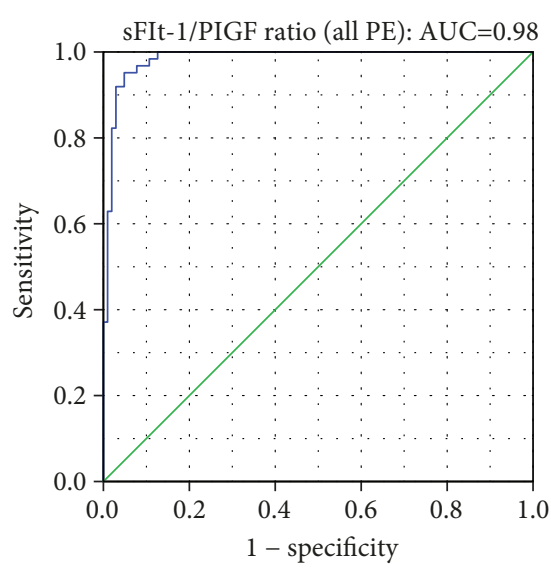

(a)

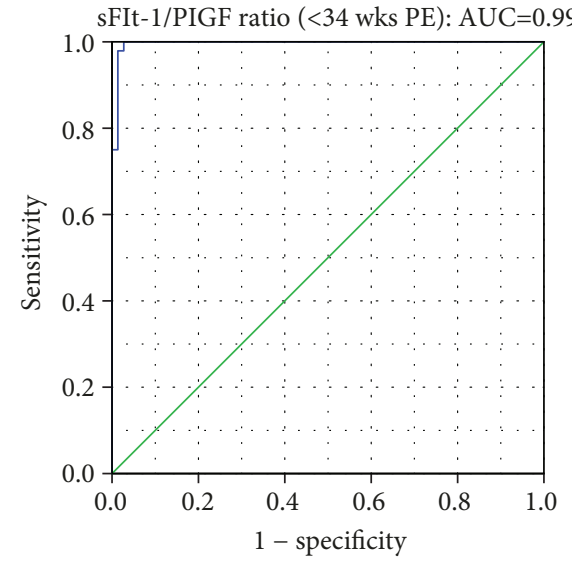

(b)

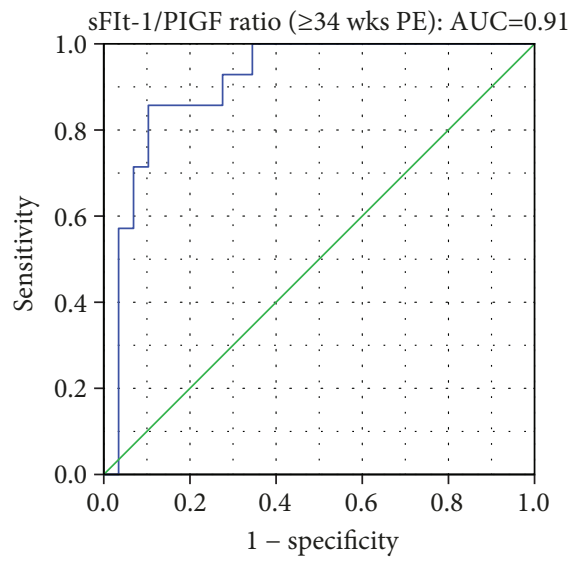

(c)

FIGURE 2: ROC curves for PE diagnosis. Receiver operating characteristic (ROC) curves of the sFlt-1/PlGF ratio were constructed in all PE $(\mathrm{AUC}=0.98,95 \% \mathrm{CI}, 0.969-1.000)$, early onset PE (AUC $=0.99,95 \% \mathrm{CI}, 0.989-1.000)$, and late onset PE (AUC $=0.91,95 \% \mathrm{CI}, 0.822-$ 1.000). When cutoff of 21.5 was used, the NPV for the diagnosis of PE was $97.9 \%, 100 \%$, and $91.7 \%$ for all, early onset, and late onset PE. When cutoff of 97.2 was used, the PPV for the diagnosis of PE was $97.5 \%, 100 \%$, and $75.0 \%$ for all, early onset, and late onset PE.

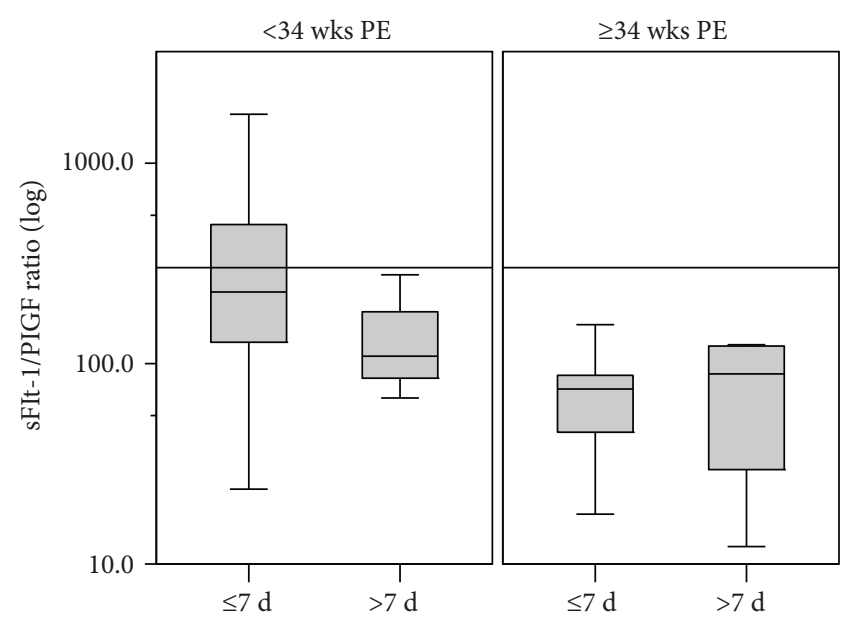

FIGURE 3: Correlation between sFlt-1/PlGF ratio and time of delivery in PE patients. Box-whisker plots displayed the logarithmic distribution of the sFlt-1/PlGF ratio in two subgroups of PE patients: delivered within $7 \mathrm{~d}(n=45)$ and longer than $7 \mathrm{~d}(n=17)$ from the time enrolled in the hospital. Boxes indicate interquartile range; whisker indicates range; error bars indicate median. $\bigcirc$ means mild outliers. * means extreme outliers. The sFlt-1/PlGF ratio of 301.0 was drawn into the graph as a solid line. The sFlt-1/PlGF ratio in the subgroup delivered within $7 \mathrm{~d}$ was significantly higher than the subgroup delivered longer than $7 \mathrm{~d}(P=0.004)$.

others will not. These patients may have similar symptoms with PE while the management and prognosis are much different. Therefore, it is essential to make correct diagnosis. However, sometimes, it is difficult to make correct differential diagnosis merely based on current diagnosis criteria. Recently, some researchers reported the use of the sFlt$1 / \mathrm{PlGF}$ ratio in the prediction and diagnosis of $\mathrm{PE}[21,23$, $33-37]$. We proved that the sFlt-1/PlGF ratio is also valuable in the diagnosis and differential diagnosis of PE in Chinese population. The sFlt-1/PlGF ratio is markedly increased in PE patient compared with GH, chrHTN, or other medical complications like autoimmune disease and uncomplicated proteinuria. And it is more favorable in diagnosing PE before 34 weeks of gestation than after 34 weeks. When the cutoff value was 97.2 , the positive predictive value for diagnosing $\mathrm{PE}$ was as high as $97.5 \%$. And the negative predictive value was $97.9 \%$ with a cutoff value of 21.5 . For the sFlt-1/PlGF ratio between 21.5 and 97.2, we agreed with the other researchers to repeat the measurement in 1 2 weeks.

Although the number of GH patients was limited in our study, we can still find the trend in this group. About 50\% $(6 / 12)$ of GH cases had sFlt-1/PlGF ratios less than 21.5, 41.7\% (5/12) were between 27.5 and 97.2. All of them had smooth pregnancy outcomes. Only one GH patient born a $1750 \mathrm{~g}$ baby at $35^{+6}$ weeks by C-section, who had a high sFlt-1/PlGF ratio of 207.7 at 34 weeks. After 34 weeks of gestation, the sFlt-1/PIGF ratio difference was not significant between PE and GH. This finding is in line with Noori et al. [38] but slightly different from those of von Dadelszen et al. [36] who found significant differences between GH and $\mathrm{PE}$ in both gestational weeks' groups. Our data also showed, when PE are ruled out, $86.7 \%(13 / 15)$ of chrHTN, $68.8 \%(11 / 16)$ of CTD, $84.6 \%(11 / 13)$ of proteinuria, and 98.7\% (75/76) of normal controls had a sFlt-1/PIGF ratio less than 21.5. About 25\% (11/44) of other medical complication had slightly higher ratio than 21.5 but none exceed 97.2 ; for these women, repeating the test in 1 2 weeks is also prudent.

Studies have shown that the risk, clinical manifestations, and severity of PE are related to the maternal race and ethnicity $[39,40]$. For instance, compared to non-Hispanic white women, there was a slightly decreased risk for East Asian women and the highest risk for Mexican women. Those likely reflected a difference in the mechanism of hypertensive disease among different ethnic women. That was why cutoff values and predictive efficacy of the sFlt-1/PlGF ratio varied largely across study countries [23-26]. We conducted a study in Chinese women, clarified its value in diagnosis of PE and prediction of pregnancy duration, and proposed a cutoff value suitable for Chinese population first time. In addition, 
the very pregnancies with several internal medical complications were included in our study. Owing to the overlap about clinical manifestations, it is difficult to differentiate these diseases from PE sometimes. Our study indicated that the sFlt$1 / \mathrm{PlGF}$ ratio may be a workable serological marker for the differential diagnosis. At present, no similar reports have been found and the value deserves further study.

4.2. sFlt-1/PlGF Ratio as a Potential Indicator of Remaining Duration of Pregnancy in PE. Predicting the delivery time is crucial for timely administration of glucocorticoid for fetal lung maturation and transferring of the patients to the medical institution with NICU. Elevated blood pressure and proteinuria are the two most important clinical indexes in the diagnosis of PE, but they are far from satisfying to provide information about the clinical course of the disease or maternal and fetal adverse outcomes [41]. Verlohren [35] showed that the sFlt-1/PlGF ratio was correlated with the remaining pregnancy duration, thus allowing individualized risk stratification for an imminent delivery of PE patients. We supported their conclusion and found in our study that the sFlt-1/PlGF ratio in PE patients whose remaining pregnancy duration was within $7 \mathrm{~d}$ was significantly higher than those with more than $7 \mathrm{~d}$ especially in severe or early onset PE patients. All patients whose ratios were higher than 301.0 (Q3, the third quartile of the sFlt-1/PlGF ratio in early onset $\mathrm{PE}$ patients) were delivered within $7 \mathrm{~d}$ regardless of disease severity, while only $66.0 \%$ (33/50) of patients whose ratios were lower than 301.0 were delivered within $7 \mathrm{~d}(P=0.018)$. These data have important clinical implication. It provides an independent classification method to the stratification of $\mathrm{PE}$ patients besides clinical criteria [42].

\section{Conclusions}

The serum sFlt-1/PlGF ratio is an efficient marker in the diagnosis and differential diagnosis of preeclampsia. This ratio can also be used to predict the timing of delivery for preeclampsia patients.

\section{Data Availability}

The data used to support the findings of this study are available from the corresponding author upon request.

\section{Conflicts of Interest}

No conflict of interest exists.

\section{Acknowledgments}

This study was funded by CAMS Innovation Fund for Medical Sciences (CIFMS) (2017-I2M-3-007).

\section{References}

[1] K. S. Khan, D. Wojdyla, L. Say, A. M. Gülmezoglu, and P. F. A. van Look, "WHO analysis of causes of maternal death: a systematic review," The Lancet, vol. 367, no. 9516, pp. 10661074, 2006.
[2] L. Duley, "The global impact of pre-eclampsia and eclampsia," Seminars in Perinatology, vol. 33, no. 3, pp. 130-137, 2009.

[3] C. H. Backes, K. Markham, P. Moorehead, L. Cordero, C. A. Nankervis, and P. J. Giannone, "Maternal preeclampsia and neonatal outcomes," Journal of Pregnancy, vol. 2011, Article ID 214365, 7 pages, 2011.

[4] B. Jim and S. A. Karumanchi, "Preeclampsia: pathogenesis, prevention, and long-term complications," Seminars in Nephrology, vol. 37, no. 4, pp. 386-397, 2017.

[5] L. Ghulmiyyah and B. Sibai, "Maternal mortality from preeclampsia /eclampsia," Seminars in Perinatology, vol. 36, no. 1, pp. 56-59, 2012.

[6] H. Stepan, I. Herraiz, D. Schlembach et al., "Implementation of the sFlt-1/PIGF ratio for prediction and diagnosis of pre-eclampsia in singleton pregnancy: implications for clinical practice," Ultrasound in Obstetrics \& Gynecology, vol. 45, no. 3, pp. 241-246, 2015.

[7] J. M. Roberts, R. N. Taylor, T. J. Musci, G. M. Rodgers, C. A. Hubel, and M. K. McLaughlin, "Preeclampsia: an endothelial cell disorder," American Journal of Obstetrics and Gynecology, vol. 161, no. 5, pp. 1200-1204, 1989.

[8] E. Phipps, D. Prasanna, W. Brima, and B. Jim, "Preeclampsia: updates in pathogenesis, definitions, and guidelines," Clinical Journal of the American Society of Nephrology, vol. 11, no. 6, pp. 1102-1113, 2016.

[9] Z. Armaly, J. E. Jadaon, A. Jabbour, and Z. A. Abassi, "Preeclampsia: novel mechanisms and potential therapeutic approaches," Frontiers in Physiology, vol. 9, p. 973, 2018.

[10] S. Sohlberg, A. Mulic-Lutvica, P. Lindgren, F. Ortiz-Nieto, A. K. Wikström, and J. Wikström, "Placental perfusion in normal pregnancy and early and late preeclampsia: a magnetic resonance imaging study," Placenta, vol. 35 , no. 3, pp. $202-$ 206, 2014.

[11] H. W. Yung, D. Atkinson, T. Campion-Smith, M. Olovsson, D. S. Charnock-Jones, and G. J. Burton, "Differential activation of placental unfolded protein response pathways implies heterogeneity in causation of early- and late-onset pre-eclampsia," The Journal of Pathology, vol. 234, no. 2, pp. 262-276, 2014.

[12] G. J. Burton and E. Jauniaux, "Placental oxidative stress: from miscarriage to preeclampsia," Journal of the Society for Gynecologic Investigation, vol. 11, no. 6, pp. 342-352, 2004.

[13] R. B. Ness and J. M. Roberts, "Heterogeneous causes constituting the single syndrome of preeclampsia: a hypothesis and its implications," American Journal of Obstetrics and Gynecology, vol. 175 , no. 5, pp. 1365-1370, 1996.

[14] N. Sattar and I. A. Greer, "Pregnancy complications and maternal cardiovascular risk: opportunities for intervention and screening?," BMJ, vol. 325, no. 7356, pp. 157-160, 2002.

[15] C. W. Redman and I. L. Sargent, "Latest advances in understanding preeclampsia," Science, vol. 308, no. 5728, pp. 1592 1594, 2005.

[16] H. Hagmann, R. Thadhani, T. Benzing, S. A. Karumanchi, and H. Stepan, "The promise of angiogenic markers for the early diagnosis and prediction of preeclampsia," Clinical Chemistry, vol. 58, no. 5, pp. 837-845, 2012.

[17] T. Cindrova-Davies, "Gabor Than Award Lecture 2008: pre-eclampsia - from placental oxidative stress to maternal endothelial dysfunction," Placenta, vol. 30, pp. 55-65, 2009.

[18] M. Widmer, J. Villar, A. Benigni, A. Conde-Agudelo, S. A. Karumanchi, and M. Lindheimer, "Mapping the theories of preeclampsia and the role of angiogenic factors: a systematic 
review," Obstetrics and Gynecology, vol. 109, no. 1, pp. 168$180,2007$.

[19] S. E. Maynard, J. Y. Min, J. Merchan et al., "Excess placental soluble fms-like tyrosine kinase 1 (sFlt1) may contribute to endothelial dysfunction, hypertension, and proteinuria in preeclampsia," The Journal of Clinical Investigation, vol. 111, no. 5, pp. 649-658, 2003.

[20] R. J. Levine, S. E. Maynard, C. Qian et al., "Circulating angiogenic factors and the risk of preeclampsia," The New England Journal of Medicine, vol. 350, no. 7, pp. 672-683, 2004.

[21] H. Zeisler, E. Llurba, F. Chantraine et al., "Predictive value of the sFlt-1:PlGF ratio in women with suspected preeclampsia," The New England Journal of Medicine, vol. 374, no. 1, pp. 1322, 2016.

[22] U. Sovio, F. Gaccioli, E. Cook, M. Hund, D. S. Charnock-Jones, and G. C. S. Smith, "Prediction of preeclampsia using the soluble fms-like tyrosine kinase 1 to placental growth factor ratio: a prospective cohort study of unselected nulliparous women," Hypertension, vol. 69, no. 4, pp. 731-738, 2017.

[23] S. Verlohren, I. Herraiz, O. Lapaire et al., "New gestational phase-specific cutoff values for the use of the soluble fms-like tyrosine kinase-1/placental growth factor ratio as a diagnostic test for preeclampsia," Hypertension, vol. 63, no. 2, pp. 346352, 2014.

[24] J. Schiettecatte, H. Russcher, E. Anckaert et al., "Multicenter evaluation of the first automated Elecsys sFlt-1 and PlGF assays in normal pregnancies and preeclampsia," Clinical Biochemistry, vol. 43, no. 9, pp. 768-770, 2010.

[25] O. Lapaire, A. Shennan, and H. Stepan, "The preeclampsia biomarkers soluble fms-like tyrosine kinase-1 and placental growth factor: current knowledge, clinical implications, and future application," European Journal of Obstetrics, Gynecology, and Reproductive Biology, vol. 151, no. 2, pp. 122-129, 2010.

[26] Y. Liu, Y. Zhao, A. Yu, B. Zhao, Y. Gao, and H. Niu, "Diagnostic accuracy of the soluble Fms-like tyrosine kinase-1/placental growth factor ratio for preeclampsia: a meta-analysis based on 20 studies," Archives of Gynecology and Obstetrics, vol. 292, no. 3, pp. 507-518, 2015.

[27] S. Rana, C. E. Powe, S. Salahuddin et al., "Angiogenic factors and the risk of adverse outcomes in women with suspected preeclampsia," Circulation, vol. 125, no. 7, pp. 911-919, 2012.

[28] American College of Obstetricians and Gynecologists and Task Force on Hypertension in pregnancy, "Hypertension in pregnancy. Report of the American College of Obstetricians and Gynecologists' task force on hypertension in pregnancy," Obstetrics and Gynecology, vol. 122, no. 5, pp. 1122-1131, 2013.

[29] A. L. Tranquilli, G. Dekker, L. Magee et al., "The classification, diagnosis and management of the hypertensive disorders of pregnancy: a revised statement from the ISSHP," Pregnancy Hypertension: An International Journal of Women's Cardiovascular Health, vol. 4, no. 2, pp. 97-104, 2014.

[30] L. A. Magee, A. Pels, M. Helewa, E. Rey, P. von Dadelszen, and Hypertension Guideline Committee, "Diagnosis, evaluation, and management of the hypertensive disorders of pregnancy: executive summary," Journal of Obstetrics and Gynaecology Canada, vol. 36, no. 5, pp. 416-438, 2014.

[31] S. A. Lowe, L. Bowyer, K. Lust et al., "The SOMANZ guidelines for the management of hypertensive disorders of pregnancy 2014," The Australian \& New Zealand Journal of Obstetrics \& Gynaecology, vol. 55, no. 1, pp. 11-16, 2015.
[32] Royal College of Obstetricians and Gynecologists, Hypertension in pregnancy: diagnosis and management. National Institute for Health and Clinical Excellence (2011) Clinical Guideline [CG107], National Institute for Health and Clinical Excellence, London, 2015.

[33] S. Verlohren, A. Galindo, D. Schlembach et al., "An automated method for the determination of the sFlt-1/PIGF ratio in the assessment of preeclampsia," American Journal of Obstetrics and Gynecology, vol. 202, no. 2, pp. 161.e1-161.e11, 2010.

[34] C. E. Kleinrouweler, M. M. Wiegerinck, C. Ris-Stalpers et al., "Accuracy of circulating placental growth factor, vascular endothelial growth factor, soluble fms-like tyrosine kinase 1 and soluble endoglin in the prediction of pre-eclampsia: a systematic review and meta-analysis," BJOG: An International Journal of Obstetrics and Gynaecology, vol. 119, no. 7, pp. 778-787, 2012.

[35] S. Verlohren, I. Herraiz, O. Lapaire et al., "The sFlt-1/PlGF ratio in different types of hypertensive pregnancy disorders and its prognostic potential in preeclamptic patients," American Journal of Obstetrics and Gynecology, vol. 206, no. 1, pp. 58.e1-58.e8, 2012.

[36] P. von Dadelszen, B. Payne, J. Li et al., "Prediction of adverse maternal outcomes in pre-eclampsia: development and validation of the fullPIERS model," The Lancet, vol. 377, no. 9761, pp. 219-227, 2011.

[37] T. H. Nguyen, T. C. Bui, T. M. Vo, Q. M. Tran, L. T.-T. Luu, and T. D. Nguyen, "Predictive value of the sFlt-1 and PlGF in women at risk for preeclampsia in the south of Vietnam," Pregnancy Hypertension, vol. 14, pp. 37-42, 2018.

[38] M. Noori, A. E. Donald, A. Angelakopoulou, A. D. Hingorani, and D. J. Williams, "Prospective study of placental angiogenic factors and maternal vascular function before and after preeclampsia and gestational hypertension," Circulation, vol. 122, no. 5, pp. 478-487, 2010.

[39] J. Gong, D. A. Savitz, C. R. Stein, and S. M. Engel, "Maternal ethnicity and preeclampsia in New York City, 1995-2003," Paediatric and Perinatal Epidemiology, vol. 26, no. 1, pp. 4552, 2012.

[40] A. A. Goodwin and B. M. Mercer, "Does maternal race or ethnicity affect the expression of severe preeclampsia?," American Journal of Obstetrics \& Gynecology, vol. 193, no. 3, pp. 973978, 2005.

[41] J. Zhang, M. A. Klebanoff, and J. M. Roberts, "Prediction of adverse outcomes by common definitions of hypertension in pregnancy," Obstetrics and Gynecology, vol. 97, no. 2, pp. 261-267, 2001.

[42] S. Rana, W. T. Schnettler, C. Powe et al., "Clinical characterization and outcomes of preeclampsia with normal angiogenic profile," Hypertension in Pregnancy, vol. 32, no. 2, pp. 189$201,2013$. 


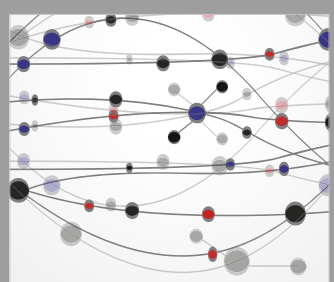

The Scientific World Journal
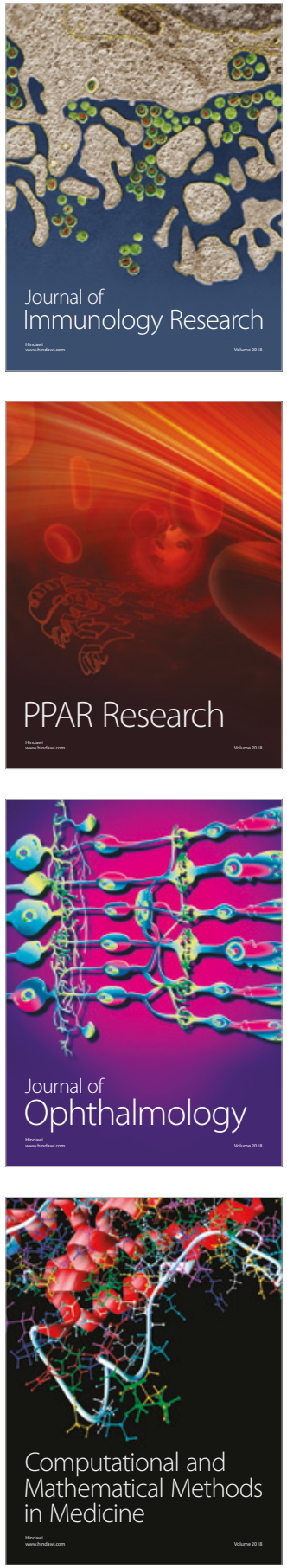

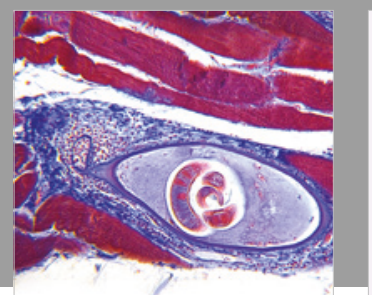

Gastroenterology Research and Practice

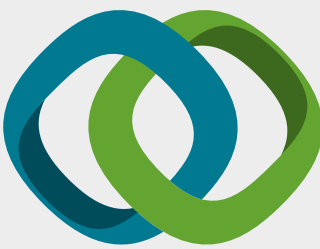

\section{Hindawi}

Submit your manuscripts at

www.hindawi.com
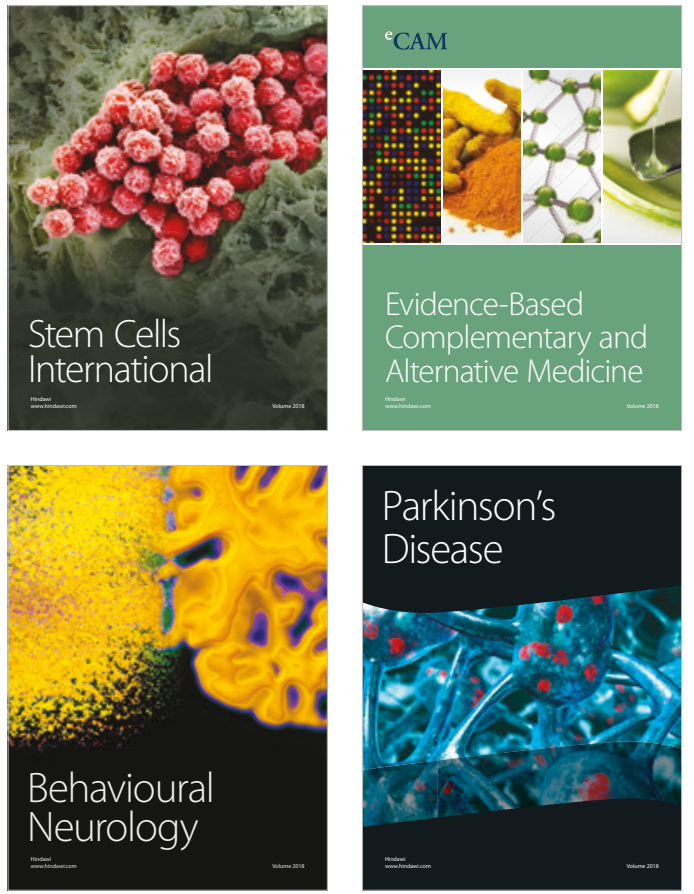

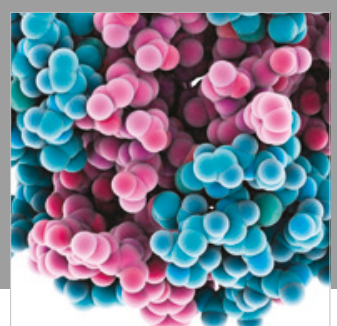

ournal of

Diabetes Research

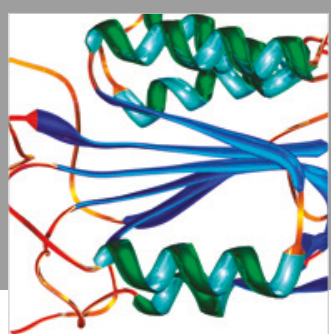

Disease Markers
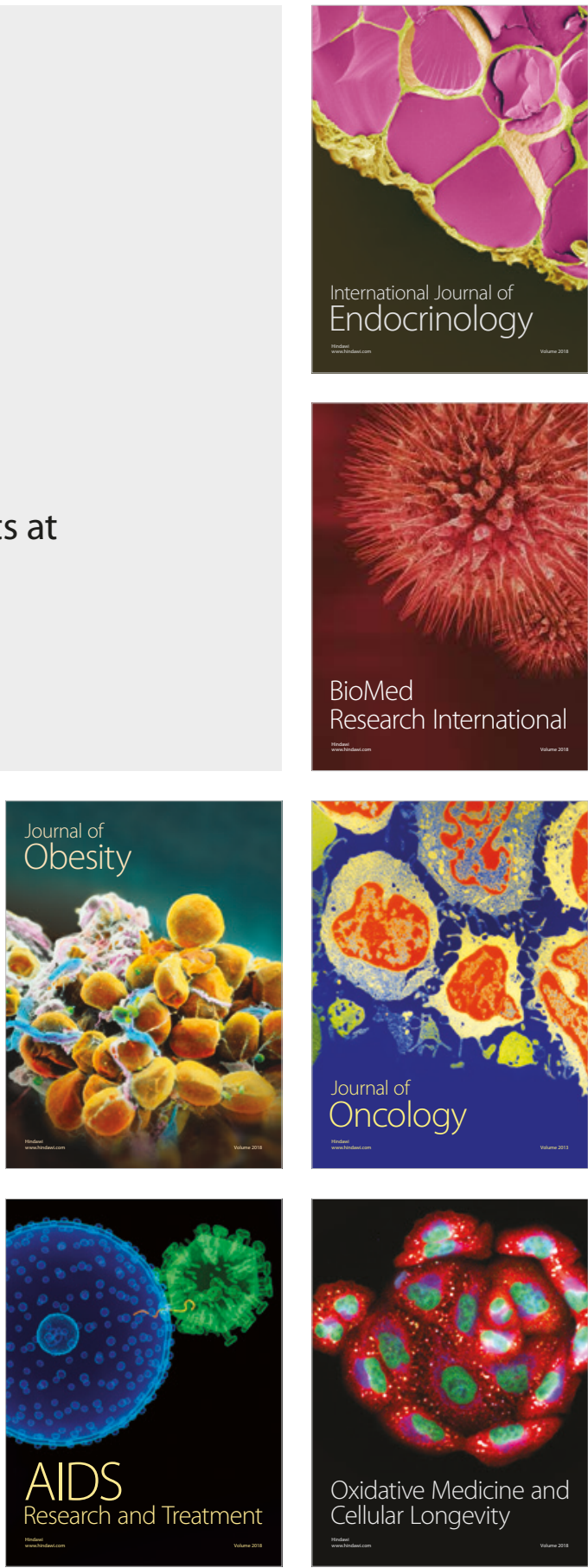